\title{
Apply activity-based costing to calculate product cost in small and medium enterprises
}

\author{
Tandung Huynh ${ }^{1,2}$, Guangming Gong ${ }^{2}$, Ngocminh $\mathrm{Ngo}^{3}$ \\ ${ }^{1}$ Business School, Hunan University, China, Faculty of Accounting and Auditing in Industrial University of Hochiminh City, Vietnam \\ ${ }^{2}$ Business School, Hunan University, China \\ ${ }^{3}$ Business and Trade School, Hunan University, China, Faculty of Business Administration in Industrial University of Hochiminh City, \\ Vietnam
}

\section{Email address:}

huynhtandung_hnu@yahoo.com(T. Huynh)

\section{To cite this article:}

Tandung Huynh, Guangming Gong, Ngocminh Ngo. Apply Activity-Based Costing to Calculate Product Cost in Small and Medium Enterprises. International Journal of Business and Economics Research. Vol. 2, No. 3, 2013, pp. 59-68.

doi: 10.11648/j.ijber.20130203.13

\begin{abstract}
Traditional costing (TDC) has been used many decades and very popular with organizations because it is simple and inexpensive. In today's business environment, overhead cost has a significant proportion in total cost, using TDC for assigning overhead cost lead to inaccuracy of product cost. Activity-Based Costing (ABC), until now, is considered as a modern costing method that overcomes the limitations of TDC and it has been popular in developed countries. However, it is until new method in developing countries, so it needs to be more diffused in this countries. This paper conducted the project on ABC method in Van Chinh enterprise in Vietnam. We defined ten activities centers as shown in table 4. Then, we applied $\mathrm{ABC}$ method to calculate cost of products and compared with the results calculated by TDC method. This paper concluded that $\mathrm{ABC}$ method is practical and appropriate for such a Van Chinh enterprise and provides more accurate information for cost management and management decision-making.
\end{abstract}

Keywords: Activity-Based Costing (ABC), Traditional Costing (TDC), Vietnam, Van Chinh Enterprise, Cost Analysis, Small and Medium Enterprises

\section{Introduction}

Traditional costing systems lead to large distortions in reporting the cost of products, services, and customers. Based on its information, managers may make serious mistakes in decision-making. It is needed to find another costing method in order to cover the TDC limitations. In the mid 1980s, the other costing method with named Activity-Based Costing (ABC) was developed by Kaplan, and it has promptly applied very popular in developed countries with obvious advantages. Several surveys conducted in developed and developing countries showed that $\mathrm{ABC}$ systems brought many advantages and benefits for organizations (see Krumwiede 1998, Nassar et al (2011), Cohen et al (2005), Baird (2007), Khozein and Dankoob (2011), Zhang and Isa (2010), Chen at el (1993)). Today more and more developing countries such as china, Thailand, Malaysia, Morocco, etc have adopted and implemented it. The prior literature presented that $\mathrm{ABC}$ application in developing counties also brought many advantages over TDC (see Liu and Pan (2007), Zhang and Isa (2010), Chongruksut and Brooks (1993)). The core of
ABC method not only accurately calculates the cost of goods (products or services) but also helps the organizations definite value-added activities and non-value-added activities and support credible information for managers to make good decisions. In order to accomplish this mission, ABC must assign accurately indirect cost to products. By using multiple drivers to assign indirect cost, $\mathrm{ABC}$ has achieved its mission and it is perceived as a normal costing method by researchers, academics as well as the accounting practitioners. ABC was developed to provide more accurate ways of assigning the costs of indirect and support resources to activities, business process, products, services, and customers.

The main difference between $\mathrm{ABC}$ and TDC is assign indirect costs to products for calculating product cost. Indirect costs incurred in organization are caused by its activities and different type of indirect cost has a different relationship with products. How can $\mathrm{ABC}$ assign accurately indirect costs to products? In order to do this, it must assign indirect cost based on cause-and-effect relationship with products. This is the basic theory to construct the $\mathrm{ABC}$ method. Therefore, ABC must use multiple cost drivers to reflect and measure indirect costs. In contrast, TDC assigns 
them by using only one cost driver such as direct-labor hours or machine hours so it leads to distort cost information. Kaplan (1998) presented the goal of ABC is not only to allocate common costs to products but its goal is also to measure and then price out all the resources used for activities that support the production and delivery of products and service to customers.

Although $\mathrm{ABC}$ has numerous advantages over TDC, but still has disadvantages that make it difficult to apply. From prior literatures we can summarize its disadvantages as following: the first in order to construct the $\mathrm{ABC}$ model, the companies must collect and analyze costs of many different activities, the second is that $\mathrm{ABC}$ needs more cost-drivers than TDC, the identification of cost drivers is often complex, the third $\mathrm{ABC}$ requires the personnel ability of employees and managers (Kaplan and Anderson (2004)). Other reason created obstacle for $\mathrm{ABC}$ adoption and implementation are satisfaction with the existing costing system, $\mathrm{ABC}$ implementation being associated with high costs, lack of time to undertake an assessment of $\mathrm{ABC}$ implementation, ABC's perceived inadequacy to provide more accurate cost information, lack of management support or interest and, the lack of local consultants and the high cost of consultants, finally, the requirement to follow the parent company's directives, including the selection of cost accounting systems (See Nassar et al (2011), Cohen et al (2005)).

\section{The Need of Doing Research}

From the review of prior literature, many researches on $\mathrm{ABC}$ method were done developed countries and very little research has been done in developing countries. A majority of $\mathrm{ABC}$ research related to large companies, and a very small proportion related to small and medium enterprise.

From the diffusion of $\mathrm{ABC}, \mathrm{ABC}$ very popular in developed counties but it is a new method in developing countries, especially in Asian context. In Vietnam, ABC has not been applied in Vietnamese companies. According to Truong and Dinh (2009), ABC method to modern management has been widely applied in many businesses around the world, like UK, USA, Thailand, etc., but this method has not been applied in Vietnam enterprises. Huynh at el (2013) surveyed 339 Vietnamese companies, presented that there was no Vietnamese companies applied ABC method for calculating its product cost.

From academic perspective, ABC method has many advantages than TDC, thus it should be continued to study in order to innovate its limitations and foster its implementation. TDC lead to large distortions in reporting the cost of activities, processes, products, services, and customers. Consequently, managers may make serious mistakes in decisions made on basis of this information. $\mathrm{ABC}$ system is more detailed and more accurate than a functional-based cost management system. $\mathrm{ABC}$ is the core of $\mathrm{ABM}$ - the new cost management system might be more accurately referred to as an activity-and-strategic-based cost management system. ABC systems not only develop more accurate costs, they also aid control costs. Because
$\mathrm{ABC}$ systems also focus on activities, they are a very useful tool in cost management systems. Activity-based management (ABM) is using the output of an activity-based cost accounting system to aid strategic decision making and to improve operational control of an organization. In the broadest terms, $\mathrm{ABM}$ aims to improve the value received by customers and to improve profits by identifying opportunities for improvements in strategy and operations.

So, this research applied $\mathrm{ABC}$ method to calculate product cost in Van Chinh enterprise as an illustration for an application $\mathrm{ABC}$ in SMEs. The results of this research also contributed to literature about the differences between $\mathrm{ABC}$ and TDC. Finally, this research contributed to diffuse $\mathrm{ABC}$ in the developing counties; in this case it was conducted in Vietnam.

\section{Research Methodology}

The research process of this paper includes two phases. The first phase, we study ABC theory and its practice in organization. The methodology used is a type of theoretical mining and logical reasoning to explore the advantages and benefits of $\mathrm{ABC}$ method by comparing with TDC method. Analysis and synthesis is used to review the prior literature related to our research's aims, and then the authors discuss and select the papers that are most suitable for our research to refer. The second phase, we do action research by applying $\mathrm{ABC}$ method to calculate product cost in a SMEs as an illustration for advantages of ABC method. Then, we compare the cost and profit of each product with the results calculated by TDC method. Finally, the approval method is used to confirm with Van Chinh managers about the results of $\mathrm{ABC}$ implementation after our project team left this company.

\section{Activity-Based Costing}

Under ABC method, organization operating was divided to activities, and the activities consume resources and products consume activities. Thus ABC model includes two stages: in the first stage, cost of resources is assigned to activities by direct tracing or using driver tracing, in the second stage, cost of activities is assigned to products. The basic model of $\mathrm{ABC}$ system is shown on the figure 1 :

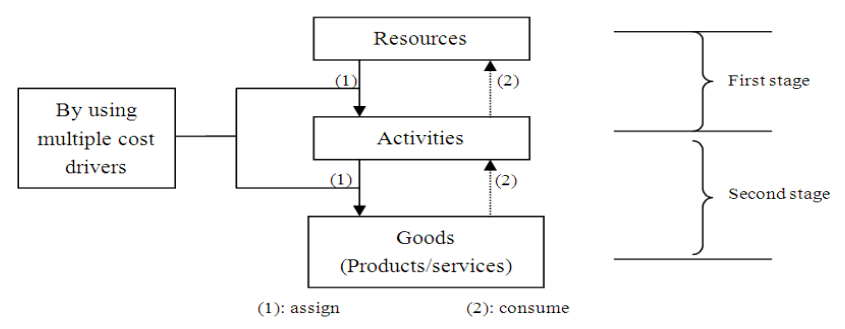

Source: Adapted from Kaplan and Atkinson, 1998

Figure 1. Basic Activity-Based Costing system

In contrast with $\mathrm{ABC}$, $\mathrm{TDC}$ use single driver for assigning indirect cost. The TDC system is shown on the figure 2 . 


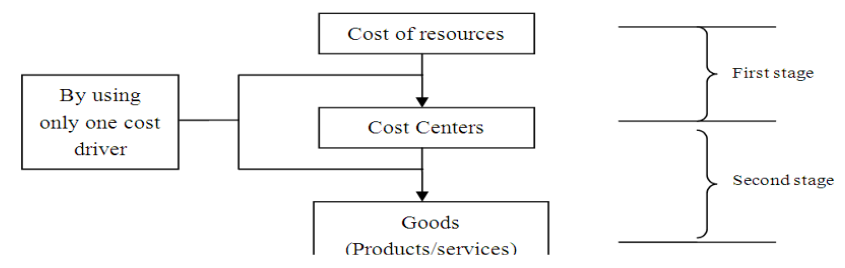

Source: Adapted from Kaplan and Atkinson, 1998

Figure 2. Traditional Costing system

We can summarize the core differences between $\mathrm{ABC}$ system and TDC system: $\mathrm{ABC}$ uses vary cost drivers for tracing indirect cost, it focus on indirect costs with cause-and-effect, and focus on the activities or processes rather than on the structure. These differences are also ABC advantages. ABC method increases the accuracy of product costs in order to increase management to a high level of confidence in cost-based decision. The comparison between ABC method and the TDC method was done prior researchers such as Kaplan (1998), Hansen (2003), Horngren (2007) to find the same and the difference between both systems. According to Kaplan (1988) traditional cost systems has simple two stages structure as shown in the figure 2 . In the first stage, service department costs are assigned to production or operating departments. In addition, the cots directly arising in these production departments are directly traced to these departments. Thus, after the first stage, all organizational expenses are assigned, either directly or through assignment from service departments, to production departments. In the second stage, costs are assigned from production departments to products processed through those departments. Both TDC system and $\mathrm{ABC}$ system have the simple two stage structure as shown above. The first stage of an ABC system has the same structure as traditional cost system, through instead of assigning service department resource costs to production centers, $\mathrm{ABC}$ systems assign resource cost of both production and service department to the activities performed by those resources. In $\mathrm{ABC}$ system, every cost assignment to an activity, or a product, service, or customer, should be transparent and traceable, via cause - and - effect relationships, to the demand for resources by cost object (whether an activity, product, service, or customer) (Kaplan, 1998). Horngren et al (2007) addressed that one of the most important differences between TDC systems and $\mathrm{ABC}$ systems is the extent of allocation across the value chain. TDC generally allocate only indirect production costs to the products. These are the only costs that can be added to the inventory value of a product for financial reporting purposes, and TDC often focus on simply measuring such inventory values. They normally do not allocate the cost of other value-chain functions because these are not appropriate costs to include in inventory. ABC systems, in contrast, focus on the costs that are important to decision makers. They often expand allocation of costs beyond production to processes such as design, marketing, order processing, and customer service. As a result, $\mathrm{ABC}$ systems are more complex than TDC but promise more accurate and useful costs to aid decision making. Hasen and Mowen
(2003) in a functional-based costing system, unit-based activity drivers supposedly explain the consumption of overhead by products. Sophisticated unit-based costing systems allocate fixed overhead to individual products, using fixed overhead rates, and they assign variable overhead, using variable overhead rates. From the perspective of $\mathrm{ABC}$, the variable overhead is appropriately traced to individual products because overhead consumption increases as units produced increases. However, assigning fixed overhead costs using unit-based drivers can be arbitrary and may not reflect the activities actually being consumed by the products. Many of the costs assigned in the traditional fixed overhead category are, in reality, batch-level, product-level, and facility-level costs that vary with drivers other than unit-based drivers. ABC systems improve product costing accuracy by recognizing that many of the so-called fixed overhead costs vary in proportion to changes other than production volume. By understanding what causes these costs to increase or decrease, they can be traced to individual products. This cause-and-effect relationship allows managers to improve product costing accuracy, which can significantly improve decision making. Additionally, this large pool of fixed overhead costs is no longer so mysterious. Knowing the underlying behavior of many these costs allows managers to exert more control over the activities that cause the costs. It also allows managers to identify which of the activities and value and which do not. Value analysis is the heart of $\mathrm{ABM}$ and is the basis for continuous improvement.

\section{Apply ABC to Calculate Product Cost in Van Chinh Enterprise}

Van Chinh, is a small and medium-sized enterprise, a furniture manufacturer that is located in Binh Duong province, Vietnam. It is invested and run by Korean. This enterprise produces many different wooden items; each kind has different technical characteristics and different resource consumption. This project was done by supporting of Van Chinh enterprise's manager and accountants under the agreement of director board. We had 6 months for this project is from April to September 2012. The team of this project analyzed the relationship of the resource, activities and products. Then, design the framework for an $\mathrm{ABC}$ system in this enterprise included 5 steps as follow:

Step 1: Identify, define, and classify activities and key attributes.

Step 2: Assign the cost of resources to activities.

Step 3: Identify cost objects and specify the amount of each activity consumed by specific cost object.

Step 4: Calculate primary activity rate.

Step 5: Assign activity cost to cost object.

The results calculated by the TDC and ABC in this case based on the data of accounting period in August 2012.

Van Chinh enterprise manufactures 21 products. The company works 27,539.50 direct labor-hours in August 2012. Costs for materials and labor for one unit of each product are given below: 
Table 1. Products and related direct costs

\begin{tabular}{|c|c|c|c|c|c|c|c|}
\hline & Product Code & $\begin{array}{l}\text { Quantity in } \\
\text { (Unit) }\end{array}$ & $\begin{array}{c}\text { Direct labor } \\
\text { hours per Unit }\end{array}$ & $\begin{array}{l}\text { Total Direct } \\
\text { labor hours }\end{array}$ & $\begin{array}{c}\text { Direct labor } \\
\text { per hour } \\
\text { (VND) } \\
\end{array}$ & $\begin{array}{c}\text { Material cost per } \\
\text { Unit } \\
\text { (VND) } \\
\end{array}$ & $\begin{array}{l}\text { Direct labor cost } \\
\text { per Unit } \\
\text { (VND) }\end{array}$ \\
\hline 1 & V-K & 453 & 2.00 & 906.00 & 21,000 & 680,698 & 42,000 \\
\hline 2 & V-F & 357 & 2.00 & 714.00 & 21,000 & 680,960 & 42,000 \\
\hline 3 & $\mathrm{~V}-\mathrm{T}$ & 353 & 2.00 & 706.00 & 21,000 & 705,594 & 42,000 \\
\hline 4 & V-Q & 123 & 2.00 & 246.00 & 21,000 & 588,929 & 42,000 \\
\hline 5 & V-200 & 156 & 2.50 & 390.00 & 21,000 & 689,221 & 52,500 \\
\hline 6 & V-200S & 826 & 2.50 & $2,065.00$ & 21,000 & 594,410 & 52,500 \\
\hline 7 & V-080 & 56 & 2.50 & 140.00 & 21,000 & 498,852 & 52,500 \\
\hline 8 & V-900 & 431 & 2.50 & $1,077.50$ & 21,000 & 693,852 & 52,500 \\
\hline 9 & V-900Q & 356 & 3.00 & $1,068.00$ & 21,000 & 637,948 & 63,000 \\
\hline 10 & V-208Q & 489 & 3.00 & $1,467.00$ & 21,000 & 551,144 & 63,000 \\
\hline 11 & V-200Q & 456 & 3.00 & $1,368.00$ & 21,000 & 623,523 & 63,000 \\
\hline 12 & V-1010 & 145 & 3.00 & 435.00 & 21,000 & 389,043 & 63,000 \\
\hline 13 & KK & 423 & 5.00 & $2,115.00$ & 21,000 & 294,508 & 105,000 \\
\hline 14 & BTD-Q & 256 & 4.50 & $1,152.00$ & 21,000 & 420,934 & 94,500 \\
\hline 15 & BTD-K & 463 & 4.00 & $1,852.00$ & 21,000 & 439,917 & 84,000 \\
\hline 16 & BTD-S & 553 & 4.00 & $2,212.00$ & 21,000 & 445,739 & 84,000 \\
\hline 17 & BTD-F & 556 & 4.00 & $2,224.00$ & 21,000 & 468,119 & 84,000 \\
\hline 18 & TDG-Q & 458 & 4.00 & $1,832.00$ & 21,000 & 371,955 & 84,000 \\
\hline 19 & TNN & 764 & 3.50 & $2,674.00$ & 21,000 & 297,955 & 73,500 \\
\hline 20 & SF-L & 156 & 4.00 & 624.00 & 21,000 & 260,251 & 84,000 \\
\hline \multirow[t]{2}{*}{21} & SF-S & 568 & 4.00 & $2,272.00$ & 21,000 & 234,424 & 84,000 \\
\hline & Total & 8,398 & & $27,539.50$ & & & \\
\hline
\end{tabular}

Source: Data provided by Van Chinh Enterprise.

The company's manufacturing overhead costs VND984,858,209 in August 2012. Under TDC, the company has used direct labor-hours as a basis for assigning overhead cost to its products. Below we show allocations of the Van Chinh enterprise's overhead costs to the products, first using TDC based on direct labor-hours as a base, and then using $\mathrm{ABC}$ with activities as a base.

\subsection{Traditional Costing Based on Direct Labor-Hours as a Base}

The company's overhead rate will be VND35,761.66 per hour are used as a base for assigning overhead costs. This rate is computed as follows:

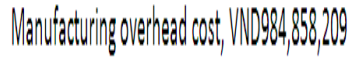

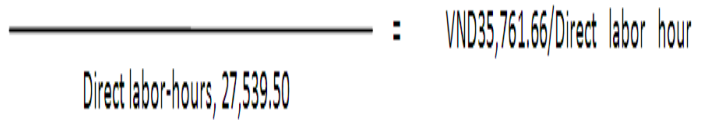

Total manufacturing cost assigned for each product as follow:
Table 2. Overhead cost assigned for products

\begin{tabular}{clrr}
\hline & Product Code & Unit & \multicolumn{1}{c}{ Total } \\
\hline 1 & V-K & 71,523 & $32,400,063$ \\
2 & V-F & 71,523 & $25,533,825$ \\
3 & V-T & 71,523 & $25,247,731$ \\
4 & V-Q & 71,523 & $8,797,368$ \\
5 & V-200 & 89,404 & $13,947,047$ \\
6 & V-200S & 89,404 & $73,847,826$ \\
7 & V-080 & 89,404 & $5,006,632$ \\
8 & V-900 & 89,404 & $38,533,188$ \\
9 & V-900Q & 107,285 & $38,193,452$ \\
10 & V-208Q & 107,285 & $52,462,354$ \\
11 & V-200Q & 107,285 & $48,921,950$ \\
12 & V-1010 & 107,285 & $15,556,322$ \\
13 & KK & 178,808 & $75,635,909$ \\
14 & BTD-Q & 160,927 & $41,197,431$ \\
15 & BTD-K & 143,047 & $66,230,593$ \\
16 & BTD-S & 143,047 & $79,104,790$ \\
17 & BTD-F & 143,047 & $79,533,930$ \\
18 & TDG-Q & 143,047 & $65,515,359$ \\
19 & TNN & 125,166 & $95,626,676$ \\
20 & SF-L & 143,047 & $22,315,275$ \\
21 & SF-S & 143,047 & $81,250,489$ \\
& Total & & $984,858,209$ \\
\hline & & & \\
\hline
\end{tabular}

Source: Data provided by Van Chinh Enterprise. 
Using this rate, the cost to one unit of each product is given below:

Table 3. Product costs calculated under TDC

\begin{tabular}{rlcccc}
\hline & Product Code & Direct materials & Direct labor & Overhead & Total cost to per unit \\
\hline 1 & V-K & 680,698 & 42,000 & 71,523 & 71,523 \\
2 & V-F & 680,960 & 42,000 & 71,523 & 794,221 \\
3 & V-T & 705,594 & 42,000 & 71,523 & 819,117 \\
4 & V-Q & 588,929 & 42,000 & 89,404 & 702,452 \\
5 & V-200 & 689,221 & 52,500 & 89,404 & 831,125 \\
6 & V-200S & 594,410 & 52,500 & 89,404 & 736,314 \\
7 & V-080 & 498,852 & 52,500 & 89,404 & 640,756 \\
8 & V-900 & 693,852 & 52,500 & 107,285 & 835,756 \\
9 & V-900Q & 637,948 & 63,000 & 107,285 & 808,233 \\
10 & V-208Q & 551,144 & 63,000 & 721,429 \\
11 & V-200Q & 623,523 & 63,000 & 107,285 & 793,808 \\
12 & V-1010 & 389,043 & 63,000 & 107,285 & 559,328 \\
13 & KK & 294,508 & 105,000 & 178,808 & 578,316 \\
14 & BTD-Q & 420,934 & 94,500 & 160,927 & 676,361 \\
15 & BTD-K & 439,917 & 143,047 & 666,964 \\
16 & BTD-S & 445,739 & 84,000 & 143,047 & 672,786 \\
17 & BTD-F & 468,000 & 143,047 & 695,166 \\
18 & TDG-Q & 371,955 & 84,000 & 143,047 & 599,002 \\
19 & TNN & 297,955 & 84,000 & 125,166 & 496,621 \\
20 & SF-L & 260,251 & 73,500 & 143,047 & 487,298 \\
21 & SF-S & 234,424 & 84,000 & 143,047 & 461,471 \\
\hline
\end{tabular}

Source: Data provided by Van Chinh Enterprise

The problem with this costing approach is that it looks only at labor time and does not consider the impact of other factors such as setups required, handle production, material receipt, inspections performed etc., on the overhead costs of the company. Therefore, since other factors are being ignored, and since the products require more labor time than other, it is assigned more amounts of overhead cost. This method is accurate only in those situations where other factors affecting overhead are not significant. In this case, these other factors are significant, as we shall see in following discussion.

Table 4. Activity and its cost driver

\begin{tabular}{ll}
\hline Activity & Cost Drivers \\
\hline Labor related & Labor-hours (DLH) \\
Run machine & Machine-hours (MH) \\
Set up machines & Setups hours \\
Handle production runs & Production runs \\
Production orders & Number of orders \\
Purchasing materials & Number of orders \\
Material receipts & Receipts \\
Parts administration & Part types \\
Quality inspections & Inspections \\
General factory & Machine-hours \\
\hline
\end{tabular}

Source: Author's identification

\subsection{Activity-Based Costing Based on Activities as a Base}

Next, we analyzed the Van Chinh enterprise operations and identified ten activities along with their associated cost drivers. In the stage one, we classified ten activity cost pools which fall into four broad categories: Unite level, Batch level, Product level, Facility level. In the stage two, we identified cost drivers for each activity cost pool. Then, we assigned the costs in each activity cost pool to products according to the proportion of each cost driver consumed by each product.

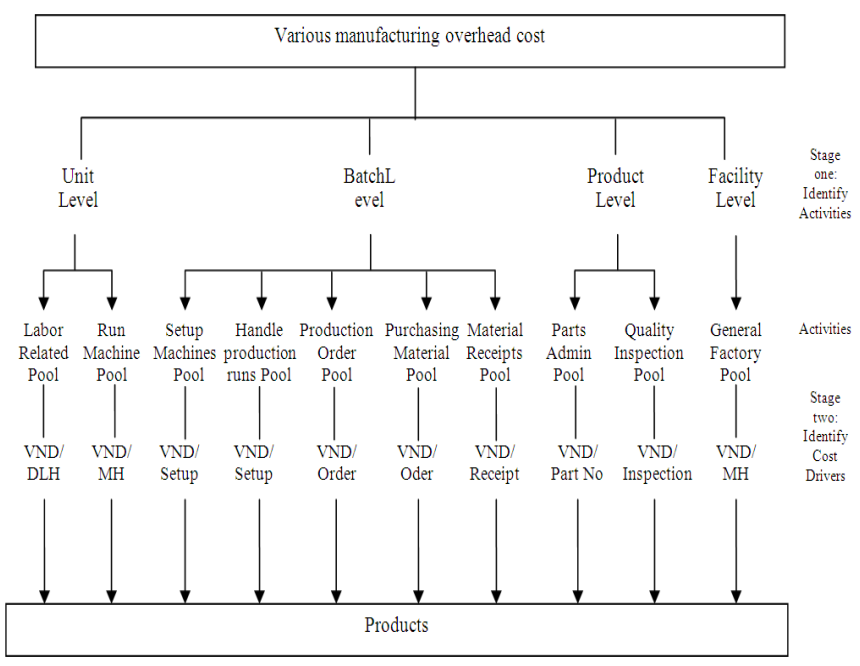

Figure 3. Activities were classified to four categories (Source: Author's identification)

The data relating to the activities are presented in Table 4.

Overhead cost was determined the amount and traced to each activity, along with the expected number of events or transactions for each center's cost driver. Data for activities are as shown in the table 5.

Using the appropriate cost drivers as a base, we computed a predetermined overhead rate for each activity.

These rates in turn have been used to assign the costs of the activity centers to the products. The results are shown on table 7 
Table 5. Overhead cost and number of transactions

\begin{tabular}{|c|c|c|c|c|c|c|c|c|c|c|c|}
\hline $\begin{array}{l}\text { Activity } \\
\text { center }\end{array}$ & $\begin{array}{l}\text { Labor } \\
\text { related }\end{array}$ & $\begin{array}{l}\text { Run } \\
\text { machin } \\
\quad \text { e }\end{array}$ & $\begin{array}{l}\text { Set up } \\
\text { machin } \\
\text { es }\end{array}$ & $\begin{array}{l}\text { Handle } \\
\text { production } \\
\text { runs }\end{array}$ & $\begin{array}{l}\text { Producti } \\
\text { on } \\
\text { orders }\end{array}$ & $\begin{array}{l}\text { Purchasi } \\
\text { ng } \\
\text { materials }\end{array}$ & $\begin{array}{l}\text { Materi } \\
\text { al } \\
\text { receipt } \\
\quad \text { s }\end{array}$ & $\begin{array}{c}\text { Parts } \\
\text { administ } \\
\text { ration }\end{array}$ & $\begin{array}{c}\text { Quality } \\
\text { inspectio } \\
\text { ns }\end{array}$ & $\begin{array}{l}\text { General } \\
\text { factory }\end{array}$ & Total \\
\hline $\begin{array}{l}\text { Traceable } \\
\text { Costs (VND) }\end{array}$ & $\begin{array}{r}194,961 \\
, 646\end{array}$ & $\begin{array}{r}162,468 \\
, 038\end{array}$ & $\begin{array}{r}110,478 \\
, 266\end{array}$ & $75,255,196$ & $\begin{array}{r}93,776,5 \\
51\end{array}$ & $\begin{array}{r}29,829,13 \\
1\end{array}$ & $\begin{array}{r}31,583 \\
786\end{array}$ & $\begin{array}{r}50,300,10 \\
5\end{array}$ & $\begin{array}{r}94,166,4 \\
75\end{array}$ & $\begin{array}{r}142,039 \\
, 015\end{array}$ & $\begin{array}{l}984,858 \\
, 209\end{array}$ \\
\hline $\begin{array}{l}\text { Total Events } \\
\text { or } \\
\text { transactions }\end{array}$ & 27,540 & 60,352 & 5,471 & 4,210 & 2,817 & 5,504 & 3,597 & 3,308 & 8,398 & 60,352 & 181,548 \\
\hline \multicolumn{12}{|c|}{ Transactions consumed by each product } \\
\hline $\mathrm{V}-\mathrm{K}$ & 906 & 960 & 82 & 75 & 50 & 71 & 86 & 40 & 453 & 960 & 3,682 \\
\hline V-F & 714 & 758 & 43 & 36 & 30 & 54 & 32 & 25 & 357 & 758 & 2,807 \\
\hline V-T & 706 & 1,024 & 73 & 49 & 37 & 72 & 58 & 36 & 353 & 1,024 & 3,432 \\
\hline V-Q & 246 & 418 & 33 & 31 & 20 & 39 & 28 & 15 & 123 & 418 & 1,371 \\
\hline V-200 & 390 & 858 & 71 & 142 & 95 & 213 & 105 & 165 & 156 & 858 & 3,053 \\
\hline V-200S & 2,065 & 4,750 & 180 & 1,200 & 960 & 1,080 & 918 & 845 & 826 & 4,750 & 17,574 \\
\hline V-080 & 140 & 126 & 12 & 12 & 12 & 14 & 21 & 13 & 56 & 126 & 532 \\
\hline V-900 & 1,078 & 862 & 33 & 31 & 24 & 60 & 43 & 29 & 431 & 862 & 3,453 \\
\hline V-900Q & 1,068 & 801 & 35 & 44 & 49 & 75 & 64 & 47 & 356 & 801 & 3,339 \\
\hline V-208Q & 1,467 & 1,306 & 79 & 72 & 55 & 135 & 105 & 49 & 489 & 1,306 & 5,063 \\
\hline V-200Q & 1,368 & 1,300 & 69 & 53 & 31 & 102 & 94 & 16 & 456 & 1,300 & 4,789 \\
\hline V-1010 & 435 & 370 & 15 & 11 & 8 & 24 & 19 & 9 & 145 & 370 & 1,405 \\
\hline KK & 2,115 & 3,173 & 368 & 245 & 164 & 369 & 158 & 62 & 423 & 3,173 & 10,250 \\
\hline BTD-Q & 1,152 & 2,995 & 298 & 199 & 132 & 297 & 152 & 130 & 256 & 2,995 & 8,606 \\
\hline BTD-K & 1,852 & 4,445 & 358 & 199 & 110 & 297 & 158 & 97 & 463 & 4,445 & 12,424 \\
\hline BTD-S & 2,212 & 6,194 & 627 & 392 & 245 & 588 & 356 & 176 & 553 & 6,194 & 17,537 \\
\hline BTD-F & 2,224 & 6,005 & 563 & 331 & 195 & 498 & 359 & 132 & 556 & 6,005 & 16,868 \\
\hline TDG-Q & 1,832 & 5,611 & 716 & 388 & 173 & 804 & 489 & 310 & 458 & 5,611 & 16,392 \\
\hline TNN & 2,674 & 8,851 & 994 & 471 & 347 & 327 & 178 & 690 & 764 & 8,851 & 24,147 \\
\hline SF-L & 624 & 1,934 & 139 & 35 & 25 & 71 & 22 & 210 & 156 & 1,934 & 5,150 \\
\hline SF-S & 2,272 & 7,611 & 683 & 195 & 56 & 314 & 152 & 212 & 568 & 7,611 & 19,674 \\
\hline Total & 27,540 & 60,352 & 5,471 & 4,210 & 2,817 & 5,504 & 3,597 & 3,308 & 8,398 & 60,352 & 181,548 \\
\hline
\end{tabular}

Source: Author's calculation

Table 6. Overhead rates by activity

\begin{tabular}{lccc}
\hline Activity Center & Traceable Costs & Total Events or Transactions & Rate per Event or Transaction (VND) \\
\hline Labor related & $194,961,646$ & 27,540 & $7,079 / \mathrm{DLH}$ \\
Run machine & $162,468,038$ & 60,352 & $2,692 / \mathrm{MH}$ \\
Set up machines & $110,478,266$ & 5,471 & $20,193 /$ setup \\
Handle production runs & $75,255,196$ & 4,210 & $17,877 /$ Run \\
Production orders & $93,776,551$ & 2,817 & $33,285 /$ order \\
Purchasing materials & $29,829,131$ & 5,504 & $5,420 /$ order \\
Material receipts & $31,583,786$ & 3,597 & $8,781 /$ receipt \\
Parts administration & $50,300,105$ & 3,308 & $15,206 /$ part type \\
Quality inspections & $94,166,475$ & 8,398 & $11,213 /$ inspection \\
General factory & $142,039,015$ & 60,352 & $2,354 / \mathrm{MH}$ \\
Total & $984,858,209$ & & \\
\hline
\end{tabular}

Source: Author's calculation 
Table 7. Overhead cost per unit of product

\begin{tabular}{|c|c|c|c|c|c|c|c|c|c|c|c|c|c|}
\hline & $\begin{array}{l}\text { Labor } \\
\text { related }\end{array}$ & $\begin{array}{c}\text { Run } \\
\text { machi } \\
\text { ne }\end{array}$ & $\begin{array}{l}\text { Set up } \\
\text { machine } \\
\text { s }\end{array}$ & $\begin{array}{l}\text { Handle } \\
\text { producti } \\
\text { on runs }\end{array}$ & $\begin{array}{l}\text { Produc } \\
\text { tion } \\
\text { orders }\end{array}$ & $\begin{array}{c}\text { Purcha } \\
\text { sing } \\
\text { materi } \\
\text { als }\end{array}$ & $\begin{array}{l}\text { Materi } \\
\text { al } \\
\text { receipt } \\
\text { s }\end{array}$ & $\begin{array}{c}\text { Parts } \\
\text { administ } \\
\text { ration }\end{array}$ & $\begin{array}{c}\text { Qualit } \\
y \\
\text { inspect } \\
\text { ions }\end{array}$ & $\begin{array}{c}\text { Gener } \\
\text { al } \\
\text { factory }\end{array}$ & $\begin{array}{c}\text { Total } \\
\text { overhea } \\
\text { d cost } \\
\text { assigned }\end{array}$ & $\begin{array}{c}\text { Num } \\
\text { ber } \\
\text { of } \\
\text { units } \\
\text { prod } \\
\text { uced }\end{array}$ & $\begin{array}{c}\text { Overhea } \\
\text { d cost } \\
\text { per unit }\end{array}$ \\
\hline $\begin{array}{c}\text { Rate } \\
\text { per } \\
\text { Event } \\
\text { or } \\
\text { Transac } \\
\text { tion } \\
\text { (VND) }\end{array}$ & $\begin{array}{l}\text { 7,079/ } \\
\text { DLH }\end{array}$ & $\begin{array}{c}2,692 / \\
\mathrm{MH}\end{array}$ & $\begin{array}{l}20,193 / \mathrm{s} \\
\text { etup }\end{array}$ & $\begin{array}{c}17,877 / \mathrm{R} \\
\text { un }\end{array}$ & $\begin{array}{c}33,285 / \\
\text { order }\end{array}$ & $\begin{array}{l}5,420 / \\
\text { order }\end{array}$ & $\begin{array}{l}8,781 / \\
\text { receipt }\end{array}$ & $\begin{array}{c}15,206 / \\
\text { part type }\end{array}$ & $\begin{array}{c}11,213 / \\
\text { inspecti } \\
\text { on }\end{array}$ & $\begin{array}{c}2,354 / \\
\mathrm{MH}\end{array}$ & & & \\
\hline V-K & $\begin{array}{c}6,413,8 \\
87\end{array}$ & $\begin{array}{c}2,584,3 \\
27\end{array}$ & $\begin{array}{c}1,655,86 \\
1\end{array}$ & $\begin{array}{c}1,332,64 \\
2\end{array}$ & $\begin{array}{c}1,654,1 \\
47\end{array}$ & $\begin{array}{c}384,78 \\
7\end{array}$ & $\begin{array}{c}755,18 \\
3\end{array}$ & 608,224 & $\begin{array}{c}5,079,4 \\
73\end{array}$ & $\begin{array}{c}2,259,3 \\
69\end{array}$ & $\begin{array}{c}22,727,9 \\
01\end{array}$ & 453 & 50,172 \\
\hline V-F & $\begin{array}{c}5,054,6 \\
53\end{array}$ & $\begin{array}{c}2,040,5 \\
42\end{array}$ & 868,318 & 640,589 & $\begin{array}{c}993,91 \\
7\end{array}$ & $\begin{array}{c}292,65 \\
5\end{array}$ & $\begin{array}{c}280,99 \\
8\end{array}$ & 380,140 & $\begin{array}{c}4,003,0 \\
28\end{array}$ & $\begin{array}{c}1,783,9 \\
60\end{array}$ & $\begin{array}{c}16,338,8 \\
00\end{array}$ & 357 & 45,767 \\
\hline V-T & $\begin{array}{c}4,998,0 \\
18\end{array}$ & $\begin{array}{c}2,756,6 \\
16\end{array}$ & $\begin{array}{c}1,474,12 \\
1\end{array}$ & 870,009 & $\begin{array}{c}1,246,0 \\
41\end{array}$ & $\begin{array}{c}390,20 \\
7\end{array}$ & $\begin{array}{c}509,31 \\
0\end{array}$ & 547,401 & $\begin{array}{c}3,958,1 \\
76\end{array}$ & $\begin{array}{c}2,409,9 \\
94\end{array}$ & $\begin{array}{c}19,159,8 \\
93\end{array}$ & 353 & 54,277 \\
\hline V-Q & $\begin{array}{c}1,741,5 \\
19\end{array}$ & $\begin{array}{c}1,125,2 \\
59\end{array}$ & 666,383 & 561,846 & $\begin{array}{c}653,80 \\
6\end{array}$ & $\begin{array}{c}211,36 \\
2\end{array}$ & $\begin{array}{c}245,87 \\
4\end{array}$ & 228,084 & $\begin{array}{c}1,379,1 \\
95\end{array}$ & $\begin{array}{c}983,76 \\
7\end{array}$ & $\begin{array}{c}7,797,09 \\
4\end{array}$ & 123 & 63,391 \\
\hline V-200 & $\begin{array}{c}2,760,9 \\
45\end{array}$ & $\begin{array}{c}2,309,7 \\
42\end{array}$ & $\begin{array}{c}1,433,73 \\
4\end{array}$ & $\begin{array}{c}2,538,52 \\
1\end{array}$ & $\begin{array}{c}3,150,9 \\
48\end{array}$ & $\begin{array}{c}1,154,3 \\
61\end{array}$ & $\begin{array}{c}922,02 \\
6\end{array}$ & $\begin{array}{c}2,508,92 \\
3\end{array}$ & $\begin{array}{c}1,749,2 \\
22\end{array}$ & $\begin{array}{c}2,019,3 \\
11\end{array}$ & $\begin{array}{c}20,547,7 \\
33\end{array}$ & 156 & 131,716 \\
\hline V-200S & $\begin{array}{c}14,618, \\
849\end{array}$ & $\begin{array}{c}12,787, \\
036\end{array}$ & $\begin{array}{c}3,634,81 \\
8\end{array}$ & $\begin{array}{c}21,452,2 \\
87\end{array}$ & $\begin{array}{c}31,953, \\
271\end{array}$ & $\begin{array}{c}5,853,1 \\
00\end{array}$ & $\begin{array}{c}8,061,1 \\
43\end{array}$ & $\begin{array}{c}12,848,7 \\
27\end{array}$ & $\begin{array}{c}9,261,9 \\
09\end{array}$ & $\begin{array}{c}11,179, \\
171\end{array}$ & $\begin{array}{c}131,650, \\
310\end{array}$ & 826 & 159,383 \\
\hline V-080 & $\begin{array}{c}991,10 \\
8\end{array}$ & $\begin{array}{c}339,19 \\
3\end{array}$ & 242,321 & 214,523 & $\begin{array}{c}399,41 \\
6\end{array}$ & 75,874 & $\begin{array}{c}184,40 \\
5\end{array}$ & 197,673 & $\begin{array}{c}627,92 \\
6\end{array}$ & $\begin{array}{c}296,54 \\
2\end{array}$ & $\begin{array}{c}3,568,98 \\
1\end{array}$ & 56 & 63,732 \\
\hline V-900 & $\begin{array}{c}7,627,9 \\
95\end{array}$ & $\begin{array}{c}2,320,5 \\
10\end{array}$ & 666,383 & 556,545 & $\begin{array}{c}811,14 \\
3\end{array}$ & $\begin{array}{c}325,17 \\
2\end{array}$ & $\begin{array}{c}377,59 \\
2\end{array}$ & 440,962 & $\begin{array}{c}4,832,7 \\
88\end{array}$ & $\begin{array}{c}2,028,7 \\
25\end{array}$ & $\begin{array}{c}19,987,8 \\
16\end{array}$ & 431 & 46,375 \\
\hline $\begin{array}{c}\text { V-900 } \\
\text { Q }\end{array}$ & $\begin{array}{c}7,560,7 \\
41\end{array}$ & $\begin{array}{c}2,156,2 \\
98\end{array}$ & 706,770 & 782,115 & $\begin{array}{c}1,620,5 \\
47\end{array}$ & $\begin{array}{c}406,46 \\
5\end{array}$ & $\begin{array}{c}559,80 \\
2\end{array}$ & 714,663 & $\begin{array}{c}3,991,8 \\
15\end{array}$ & $\begin{array}{c}1,885,1 \\
61\end{array}$ & $\begin{array}{c}20,384,3 \\
77\end{array}$ & 356 & 57,259 \\
\hline $\begin{array}{c}\mathrm{V}-208 \\
\mathrm{Q}\end{array}$ & $\begin{array}{c}10,385 \\
400\end{array}$ & $\begin{array}{c}3,515,7 \\
62\end{array}$ & $\begin{array}{c}1,595,28 \\
1\end{array}$ & $\begin{array}{c}1,283,88 \\
7\end{array}$ & $\begin{array}{c}1,840,2 \\
84\end{array}$ & $\begin{array}{c}731,63 \\
7\end{array}$ & $\begin{array}{c}922,02 \\
6\end{array}$ & 745,074 & $\begin{array}{c}5,483,1 \\
40\end{array}$ & $\begin{array}{c}3,073,6 \\
84\end{array}$ & $\begin{array}{c}29,576,1 \\
75\end{array}$ & 489 & 60,483 \\
\hline $\begin{array}{c}\mathrm{V}-200 \\
\mathrm{Q}\end{array}$ & $\begin{array}{c}9,684,5 \\
45\end{array}$ & $\begin{array}{c}3,499,6 \\
10\end{array}$ & $\begin{array}{c}1,393,34 \\
7\end{array}$ & 948,851 & $\begin{array}{c}1,026,1 \\
13\end{array}$ & $\begin{array}{c}552,79 \\
3\end{array}$ & $\begin{array}{c}825,43 \\
3\end{array}$ & 243,290 & $\begin{array}{c}5,113,1 \\
12\end{array}$ & $\begin{array}{c}3,059,5 \\
63\end{array}$ & $\begin{array}{c}26,346,6 \\
55\end{array}$ & 456 & 57,778 \\
\hline V-1010 & $\begin{array}{c}3,079,5 \\
15\end{array}$ & $\begin{array}{c}996,04 \\
3\end{array}$ & 302,901 & 191,538 & $\begin{array}{c}254,73 \\
0\end{array}$ & $\begin{array}{c}130,06 \\
9\end{array}$ & $\begin{array}{c}166,84 \\
3\end{array}$ & 136,850 & $\begin{array}{c}1,625,8 \\
80\end{array}$ & $\begin{array}{c}870,79 \\
9\end{array}$ & $\begin{array}{c}7,755,16 \\
8\end{array}$ & 145 & 53,484 \\
\hline KK & $\begin{array}{c}14,972 \\
817\end{array}$ & $\begin{array}{c}8,541,7 \\
40\end{array}$ & $\begin{array}{c}7,431,18 \\
3\end{array}$ & $\begin{array}{c}4,385,80 \\
1\end{array}$ & $\begin{array}{c}5,443,8 \\
91\end{array}$ & $\begin{array}{c}1,999,8 \\
09\end{array}$ & $\begin{array}{c}1,387,4 \\
30\end{array}$ & 942,747 & $\begin{array}{c}4,743,0 \\
84\end{array}$ & $\begin{array}{c}7,467,6 \\
86\end{array}$ & $\begin{array}{c}57,316,1 \\
87\end{array}$ & 423 & 135,499 \\
\hline BTD-Q & $\begin{array}{c}8,155,4 \\
06\end{array}$ & $\begin{array}{c}8,062,5 \\
63\end{array}$ & $\begin{array}{c}6,017,64 \\
3\end{array}$ & $\begin{array}{c}3,551,54 \\
5\end{array}$ & $\begin{array}{c}4,408,3 \\
68\end{array}$ & $\begin{array}{c}1,609,6 \\
02\end{array}$ & $\begin{array}{c}1,334,7 \\
43\end{array}$ & $\begin{array}{c}1,976,72 \\
7\end{array}$ & $\begin{array}{c}2,870,5 \\
19\end{array}$ & $\begin{array}{c}7,048,7 \\
61\end{array}$ & $\begin{array}{c}45,035,8 \\
78\end{array}$ & 256 & 175,921 \\
\hline BTD-K & $\begin{array}{c}13,110 \\
949\end{array}$ & $\begin{array}{c}11,965, \\
973\end{array}$ & $\begin{array}{c}7,229,24 \\
9\end{array}$ & $\begin{array}{c}3,555,51 \\
8\end{array}$ & $\begin{array}{c}3,677,7 \\
49\end{array}$ & $\begin{array}{c}1,609,6 \\
02\end{array}$ & $\begin{array}{c}1,387,4 \\
30\end{array}$ & $\begin{array}{c}1,474,94 \\
3\end{array}$ & $\begin{array}{c}5,191,6 \\
03\end{array}$ & $\begin{array}{c}10,461, \\
350\end{array}$ & $\begin{array}{c}59,664,3 \\
66\end{array}$ & 463 & 128,865 \\
\hline BTD-S & $\begin{array}{c}15,659, \\
513\end{array}$ & $\begin{array}{c}16,674, \\
295\end{array}$ & $\begin{array}{c}12,661,2 \\
82\end{array}$ & $\begin{array}{c}7,005,51 \\
3\end{array}$ & $\begin{array}{c}8,152,1 \\
41\end{array}$ & $\begin{array}{c}3,186,6 \\
88\end{array}$ & $\begin{array}{c}3,126,1 \\
08\end{array}$ & $\begin{array}{c}2,676,18 \\
5\end{array}$ & $\begin{array}{c}6,200,7 \\
69\end{array}$ & $\begin{array}{c}14,577 \\
639\end{array}$ & $\begin{array}{c}89,920,1 \\
31\end{array}$ & 553 & 162,604 \\
\hline BTD-F & $\begin{array}{c}15,744, \\
465\end{array}$ & $\begin{array}{c}16,165 \\
505\end{array}$ & $\begin{array}{c}11,368,9 \\
02\end{array}$ & $\begin{array}{c}5,920,41 \\
1\end{array}$ & $\begin{array}{c}6,484,1 \\
74\end{array}$ & $\begin{array}{c}2,698,9 \\
29\end{array}$ & $\begin{array}{c}3,152,4 \\
51\end{array}$ & $\begin{array}{c}2,007,13 \\
8\end{array}$ & $\begin{array}{c}6,234,4 \\
08\end{array}$ & $\begin{array}{c}14,132 \\
826\end{array}$ & $\begin{array}{c}83,909,2 \\
10\end{array}$ & 556 & 150,916 \\
\hline TDG-Q & $\begin{array}{c}12,969, \\
362\end{array}$ & $\begin{array}{c}15,104, \\
854\end{array}$ & $\begin{array}{c}14,458,4 \\
97\end{array}$ & $\begin{array}{c}6,927,30 \\
1\end{array}$ & $\begin{array}{c}5,764,4 \\
87\end{array}$ & $\begin{array}{c}4,357,3 \\
08\end{array}$ & $\begin{array}{c}4,294,0 \\
07\end{array}$ & $\begin{array}{c}4,713,73 \\
4\end{array}$ & $\begin{array}{c}5,135,5 \\
38\end{array}$ & $\begin{array}{c}13,205, \\
543\end{array}$ & $\begin{array}{c}86,930,6 \\
30\end{array}$ & 458 & 189,805 \\
\hline TNN & $\begin{array}{c}18,930, \\
171\end{array}$ & $\begin{array}{c}23,826 \\
959\end{array}$ & $\begin{array}{c}20,072,2 \\
71\end{array}$ & $\begin{array}{c}8,425,98 \\
2\end{array}$ & $\begin{array}{c}11,553 \\
475\end{array}$ & $\begin{array}{c}1,772,1 \\
89\end{array}$ & $\begin{array}{c}1,563,0 \\
54\end{array}$ & $\begin{array}{c}10,491,8 \\
60\end{array}$ & $\begin{array}{c}8,566,7 \\
05\end{array}$ & $\begin{array}{c}20,830, \\
914\end{array}$ & $\begin{array}{c}126,033, \\
578\end{array}$ & 764 & 164,965 \\
\hline SF-L & $\begin{array}{c}4,417,5 \\
12\end{array}$ & $\begin{array}{c}5,206,3 \\
43\end{array}$ & $\begin{array}{c}2,806,88 \\
7\end{array}$ & 621,222 & $\begin{array}{c}832,11 \\
6\end{array}$ & $\begin{array}{c}384,78 \\
7\end{array}$ & $\begin{array}{c}193,18 \\
6\end{array}$ & $\begin{array}{c}3,193,17 \\
5\end{array}$ & $\begin{array}{c}1,749,2 \\
22\end{array}$ & $\begin{array}{c}4,551,6 \\
88\end{array}$ & $\begin{array}{c}23,956,1 \\
39\end{array}$ & 156 & 153,565 \\
\hline SF-S & $\begin{array}{c}16,084, \\
274\end{array}$ & $\begin{array}{c}20,488, \\
869\end{array}$ & $\begin{array}{c}13,792,1 \\
14\end{array}$ & $\begin{array}{c}3,488,55 \\
0\end{array}$ & $\begin{array}{c}1,855,7 \\
89\end{array}$ & $\begin{array}{c}1,701,7 \\
35\end{array}$ & $\begin{array}{c}1,334,7 \\
43\end{array}$ & $\begin{array}{c}3,223,58 \\
6\end{array}$ & $\begin{array}{c}6,368,9 \\
64\end{array}$ & $\begin{array}{c}17,912, \\
562\end{array}$ & $\begin{array}{c}86,251,1 \\
86\end{array}$ & 568 & 151,851 \\
\hline
\end{tabular}

Source: Author's calculation

Then we calculate the cost of per unit under ABC method and comparison with TDC as follow:

Table 8. Product cost calculated by $A B C$ and TDC

\begin{tabular}{|c|c|c|c|c|c|c|c|c|c|}
\hline & \multicolumn{4}{|c|}{$\mathbf{A B C}$} & \multicolumn{4}{|c|}{ TDC } & \multirow[b]{2}{*}{$\begin{array}{c}\mathrm{ABC} / \mathrm{TDC} \\
(\%)\end{array}$} \\
\hline & $\begin{array}{c}\text { Direct } \\
\text { materials }\end{array}$ & $\begin{array}{l}\text { Direct } \\
\text { labor }\end{array}$ & Overhead & $\begin{array}{c}\text { Total per } \\
\text { unit }\end{array}$ & $\begin{array}{c}\text { Direct } \\
\text { materials }\end{array}$ & $\begin{array}{l}\text { Direct } \\
\text { labor }\end{array}$ & Overhead & $\begin{array}{l}\text { Total per } \\
\text { unit }\end{array}$ & \\
\hline V-K & 680,698 & 42,000 & 50,172 & 772,870 & 680,698 & 42,000 & 71,523 & 794,221 & 97.31 \\
\hline V-F & 680,960 & 42,000 & 45,767 & 768,727 & 680,960 & 42,000 & 71,523 & 794,483 & 96.76 \\
\hline V-T & 705,594 & 42,000 & 54,277 & 801,871 & 705,594 & 42,000 & 71,523 & 819,117 & 97.89 \\
\hline V-Q & 588,929 & 42,000 & 63,391 & 694,320 & 588,929 & 42,000 & 71,523 & 702,452 & 98.84 \\
\hline V-200 & 689,221 & 52,500 & 131,716 & 873,437 & 689,221 & 52,500 & 89,404 & 831,125 & 105.09 \\
\hline V-200S & 594,410 & 52,500 & 159,383 & 806,293 & 594,410 & 52,500 & 89,404 & 736,314 & 109.50 \\
\hline
\end{tabular}




\begin{tabular}{|c|c|c|c|c|c|c|c|c|c|}
\hline V-080 & 498,852 & 52,500 & 63,732 & 615,084 & 498,852 & 52,500 & 89,404 & 640,756 & 95.99 \\
\hline V-900 & 693,852 & 52,500 & 46,375 & 792,727 & 693,852 & 52,500 & 89,404 & 835,756 & 94.85 \\
\hline V-900Q & 637,948 & 63,000 & 57,259 & 758,207 & 637,948 & 63,000 & 107,285 & 808,233 & 93.81 \\
\hline V-208Q & 551,144 & 63,000 & 60,483 & 674,627 & 551,144 & 63,000 & 107,285 & 721,429 & 93.51 \\
\hline V-200Q & 623,523 & 63,000 & 57,778 & 744,301 & 623,523 & 63,000 & 107,285 & 793,808 & 93.76 \\
\hline V-1010 & 389,043 & 63,000 & 53,484 & 505,527 & 389,043 & 63,000 & 107,285 & 559,328 & 90.38 \\
\hline KK & 294,508 & 105,000 & 135,499 & 535,007 & 294,508 & 105,000 & 178,808 & 578,316 & 92.51 \\
\hline BTD-Q & 420,934 & 94,500 & 175,921 & 691,355 & 420,934 & 94,500 & 160,927 & 676,361 & 102.22 \\
\hline BTD-S & 445,739 & 84,000 & 162,604 & 692,343 & 445,739 & 84,000 & 143,047 & 672,786 & 102.91 \\
\hline BTD-F & 468,119 & 84,000 & 150,916 & 703,035 & 468,119 & 84,000 & 143,047 & 695,166 & 101.13 \\
\hline TDG-Q & 371,955 & 84,000 & 189,805 & 645,760 & 371,955 & 84,000 & 143,047 & 599,002 & 107.81 \\
\hline TNN & 297,955 & 73,500 & 164,965 & 536,420 & 297,955 & 73,500 & 125,166 & 496,621 & 108.01 \\
\hline SF-L & 260,251 & 84,000 & 153,565 & 497,816 & 260,251 & 84,000 & 143,047 & 487,298 & 102.16 \\
\hline SF-S & 234,424 & 84,000 & 151,851 & 470,275 & 234,424 & 84,000 & 143,047 & 461,471 & 101.91 \\
\hline
\end{tabular}

Source: Author's calculation

The table 7 presented the use of an activity approach has resulted in overhead cost being assigned to each unit of product. These amounts are used in the table above to determine the cost to a unit of each product under $\mathrm{ABC}$ method. The results from the $\mathrm{ABC}$ system were quite different from the results using the traditional cost system shown in table 8 . We can see the figure bellow presented the difference of product cost calculated by ABC method and traditional costing method.

The profits and loss of each product was shown in table 9 as follow:

Table 9. Profit calculated by $A B C$ and TDC

\begin{tabular}{|c|c|c|c|c|c|c|}
\hline & \multirow[b]{2}{*}{$\begin{array}{c}\text { Quantity in August } \\
2012 \text { (Unit) }\end{array}$} & \multirow[b]{2}{*}{ Selling Price } & \multicolumn{2}{|c|}{ ABC } & \multicolumn{2}{|c|}{ TDC } \\
\hline & & & $\begin{array}{c}\text { Total cost per } \\
\text { unit }\end{array}$ & Profit per unit & $\begin{array}{c}\text { Total cost per } \\
\text { unit }\end{array}$ & Profit per unit \\
\hline $\mathrm{V}-\mathrm{K}$ & 453 & 782,308 & 772,870 & 9,438 & 794,221 & $(11,913)$ \\
\hline V-F & 357 & 778,593 & 768,727 & 9,866 & 794,483 & $(15,890)$ \\
\hline V-T & 353 & 808,468 & 801,871 & 6,597 & 819,117 & $(10,649)$ \\
\hline V-Q & 123 & 681,378 & 694,320 & $(12,942)$ & 702,452 & $(21,074)$ \\
\hline$V-200$ & 156 & 856,059 & 873,437 & $(17,378)$ & 831,125 & 24,934 \\
\hline V-200S & 826 & 789,329 & 806,293 & $(16,964)$ & 736,314 & 53,015 \\
\hline V-080 & 56 & 631,145 & 615,084 & 16,061 & 640,756 & $(9,611)$ \\
\hline V-900 & 431 & 822,802 & 792,727 & 30,075 & 835,756 & $(12,954)$ \\
\hline V-900Q & 356 & 793,685 & 758,207 & 35,477 & 808,233 & $(14,548)$ \\
\hline V-208Q & 489 & 703,393 & 674,627 & 28,766 & 721,429 & $(18,036)$ \\
\hline V-200Q & 456 & 789,442 & 744,301 & 45,141 & 793,808 & $(4,366)$ \\
\hline V-1010 & 145 & 552,336 & 505,527 & 46,809 & 559,328 & $(6,992)$ \\
\hline KK & 423 & 581,208 & 535,007 & 46,201 & 578,316 & 2,892 \\
\hline BTD-Q & 256 & 700,034 & 691,355 & 8,679 & 676,361 & 23,673 \\
\hline BTD-K & 463 & 677,635 & 652,782 & 24,853 & 666,964 & 10,671 \\
\hline BTD-S & 553 & 688,260 & 692,343 & $(4,084)$ & 672,786 & 15,474 \\
\hline BTD-F & 556 & 719,497 & 703,035 & 16,462 & 695,166 & 24,331 \\
\hline TDG-Q & 458 & 630,150 & 645,760 & $(15,610)$ & 599,002 & 31,148 \\
\hline TNN & 764 & 527,411 & 536,420 & $(9,010)$ & 496,621 & 30,790 \\
\hline SF-L & 156 & 499,480 & 497,816 & 1,664 & 487,298 & 12,182 \\
\hline SF-S & 568 & 472,085 & 470,275 & 1,810 & 461,471 & 10,614 \\
\hline
\end{tabular}

Source: Author's calculation

\section{Discussion}

The comparison of product cost calculated by $\mathrm{ABC}$ and TDC in figure 3 shows three significant areas: products under the cost of $\mathrm{ABC}$, products which cost almost the same, and products over the cost of $\mathrm{ABC}$.

1. The S-Curve lines below the value 100 of $y$-axis show products that cost lower than calculations by TDC. The products in this area have generated more profit that enterprise that were not visible when applying TDC. In contrast, in the area where the S-curve is located above the value 100 of $y$-axis, the real cost of the products will be greater than the cost calculated TDC, these products have made a loss but the business cannot see. This area is a hidden loss. By the aid of $\mathrm{ABC}$, managers realized the "hidden profit" or "hidden loss" area. With accurate costing information, the enterprise can redefine the output price of these products; examine pricing decisions, and can gain more competitive advantage.

2. The figure 3 shows that product cost calculated by ABC method is quite difference compared with TDC. This results lead to big difference in profit provided by $\mathrm{ABC}$ and TDC as shown on figure 4 .

3. By applying the ABC system, the Van Chinh enterprise realizes the unreasonable allocation of resources. Instead of allocating resources to produce really profitable products such as V-080, V-900, V-900Q, V-208Q,V-200Q, V-1010, KK, BTD-Q, BTD-K, BTD-F, V-K, V-F, V-T, SF-L, and SF-S (Most 
of them have the real cost is smaller than the value 100 of y-axis). This enterprise has concentrated their resources for the production of products generated loss such as V-Q, V-200, V-200-S, BTD-S, TDG-Q, and TNN (the cost of these products is higher than the value 100 of $y$-axis). The results of the unreasonable allocation of resources were affected by the inaccurate cost information that TDC provided. It distorted the cost of products, provided inaccurate information; hence the business had been misled in decision making. In this case, the product V-200S is believed to generate the most profit under TDC, so this enterprise concentrated to produce it. But with $\mathrm{ABC}$ it is the product that made a loss per unit at the second rank after product $\mathrm{V}-200$.
After applying the ABC system, managers in this enterprise have confirmed with us that, besides being able to calculate the accurate costs of products, ABC also helps the business discover many non-value-added activities that TDC does not indicate such as: the number of times saw blades were replaced, the numerous times the cutting mold had to be changed, the numerous times raw materials had to be tested and made outputs of production, the long wait it took for semi-finished goods to continue to the next step of the production process. Therefore the managers strongly believe to use information provided by $\mathrm{ABC}$ system to assist them in making appropriate decisions to improve production processes, improve efficiency, reduce costs, and pricing decisions.

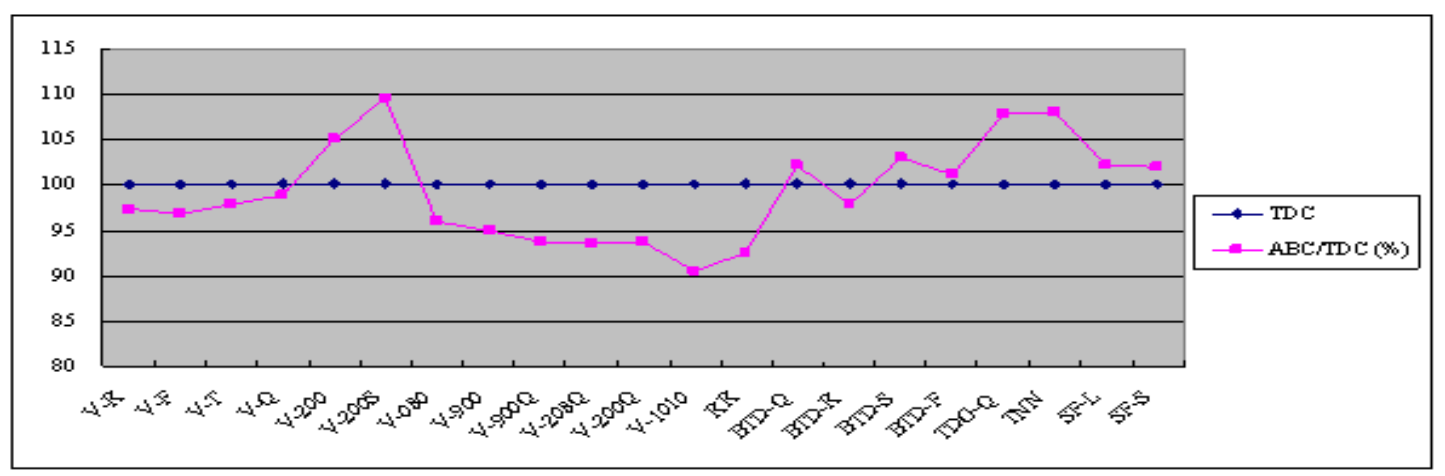

Figure 3. S-Curve (bias in percentage)

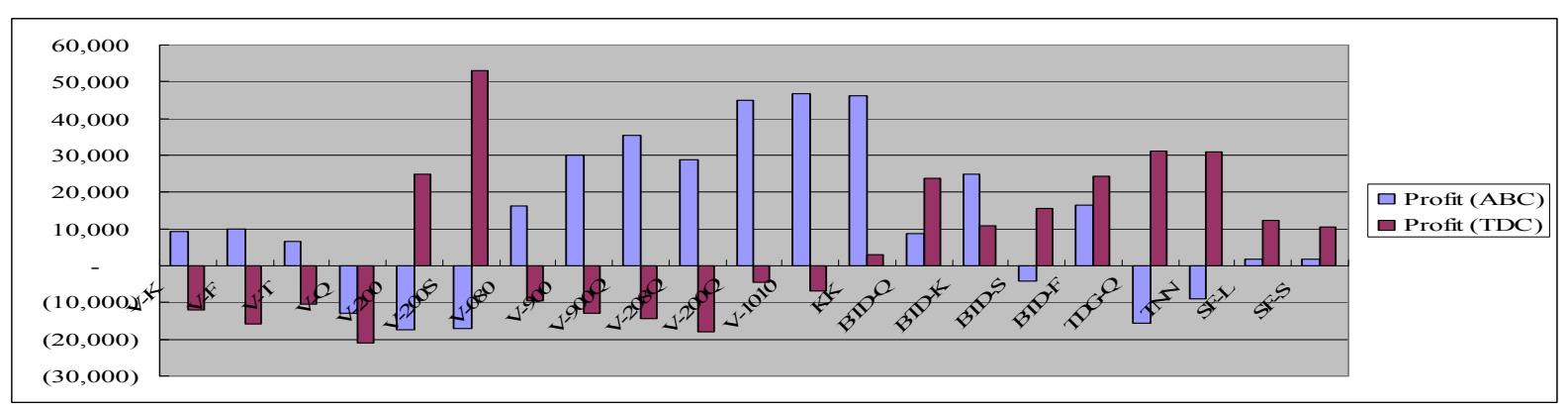

Figure 4. Comparison of profit calculated by $A B C$ and TDC

\section{Conclusions}

This research attempts design and apply $\mathrm{ABC}$ in SMEs in Vietnam as an illustration for diffusion $\mathrm{ABC}$ method. Using the data provided by Van Chinh enterprise, this paper calculated unit cost of each product. Then compare it with the result of TDC. There was the big difference between product costs calculated by two methods. Before this project were conducted, this enterprise using TDC method with one cost driver (labor hour) to assign overhead cost. The distortion of information provided by TDC leads Van Chinh's managers met many mistake in decision-making. By using ten cost drivers to assign overhead cost, the product cost calculated by $\mathrm{ABC}$ method is more accurate. $\mathrm{ABC}$ method also indicates products generate profit and products make a loss for this enterprise. Under ABC method, information provided more detailed, more confident for managers to make decision.
Beside the advantages and profits that $\mathrm{ABC}$ method has brought for Van Chinh enterprise, the application of $\mathrm{ABC}$ method has met some difficulties such as: lack of human resource, high costs, identification of cost drivers is complex. In which lack of human resource is the most difficulties. This enterprise meets difficulties to find qualified accountants that understand and have skill in $\mathrm{ABC}$ method. This is also a note for universities more emphasize to focus on management accounting especially in $\mathrm{ABC}$ method when providing accounting courses in Vietnam.

\section{Acknowledgments}

We would like to thank Van Chinh Enterprise, especially director board and accountants supported and provided for this project. We also thank Dong Su Financial Taxable Consulting Co., Ltd that offered many valuable consulting. Finally, we thank to all authors that our paper cited. 


\section{References}

[1] Chen, G.M., Firth, M. and Park, K., 1993. The implementation and benefits of activity-based costing: a Hong Kong study. Asian Review of Accounting, 9(2), 23-37.

[2] Chongruksut,. W. and Brooks, A., 1993. The adoption and implementation of Activity-Based Costing in Thailand. Asian Review of Accounting, 13(2), 1-17.

[3] Cohen, S., Venieris, G. and Kaimenaki, E., 2005. ABC: adopters, supporters, deniers and unawares. Managerial Auditing Journal, 20(9), 981-1000.

[4] Hansen, D.R. and Mowen, M.M., 2003. Cost Management: Accounting and Control. Peking: Peking University Press.

[5] Horngren, Charles T., Sundem Gary L., Stratton William O. Introduction to management accounting. Peking University Press, 2007.

[6] Kaplan, R.S. and Atkinson, A.A., 1998. Advanced Management Accounting. Prentice Hall International, Inc.

[7] Kaplan, R.S. and Anderson, S., 2004. Time-Driven Activity-Based costing. Harvard Business Review, 2004, November, 1-9.

[8] Kevin Baird, 2007. Adoption of activity management practices in public sector organizations, Accounting and Finance, 47, 551-569.

[9] Khozein, A. and Dankoob, M., 2011. Activity Based Costing System and its succeed implementing in organizations. Australian Journal of Basic and Applied Sciences, 2011, 5(10), 613-619.
[10] Krumwiede, K.R., 1998. ABC: Why it's tried and how it succeeds. Management Accounting, April: 32-38.

[11] Liu, L.Y.J. and Pan, F., 2007. The implementation of Activity-Based Costing in China: An innovation action research approach. The British Accounting Review, 39, 249-264.

[12] Nassar, M., Al-Khadash, H.A. and Sangster, A., 2011. The diffusion of activity-based costing in Jordanian industrial companies. Qualitative Research in Accounting \& Management, 8(2), 180-200.

[13] Zhang, Y.F. and Isa, C.R., 2010. Behavioral and organizational variables affecting the success of $\mathrm{ABC}$ success in China. African Journal of Business Management, 4(11), 2302-2308.

[14] Tandung Huynh, Gong Guang Ming, Tran Phuoc, 2013. So sánh phương pháp $\mathrm{ABC}$ và phương pháp tính giá truyền thống - sự cần thiết áp dụng phương pháp $\mathrm{ABC}$ cho các doang nghiệp Việt Nam. Tạp chí Đại Học Công Nghiệp thành phố Hồ Chí Minh, 10(1), 58-70. (Tandung Huynh, Gong Guang Ming, Tran Phuoc, 2013. A comparative analysis of Activity-Based Costing and traditional costing, the necessary to apply $\mathrm{ABC}$ in Vietnamese companies. Journal of Ho Chi Minh City University of Industry, 10(1), 58-70).

[15] Trương Bá Thanh, Đinh Thị Thanh Hường, 2009. Vận dụng phương pháp tính giá dựa trên cơ sở hoạt động trong các doanh nghiệp in. Tạp chí khoa học và công nghệ, đại học Đà Nẵng, 3(32), 1-10. (Truong, B.T. and Dinh, T.H., 2009. Apply $\mathrm{ABC}$ method in the printing enterprise. Journal of Da Nang Science and Technology, 3(32), 1-10). 